\title{
Avaliação bioeconômica de estratégias de alimentação em sistemas de produção de leite. 2 . Metodologia alternativa: nível de utilização de capital ${ }^{1}$
}

\author{
Francisco Palma Rennó ${ }^{2}$, José Carlos Pereira ${ }^{3}$, Carlos Antônio Moreira Leite ${ }^{4}$, Marcelo \\ Teixeira Rodrigues ${ }^{3}$, Oriel Fajardo de Campos $^{5}$, Dilermando Miranda da Fonseca ${ }^{3}$, \\ Luciana Navajas Rennó ${ }^{6}$
}

\author{
1 Pesquisa parcialmente financiada pelo CNPq. \\ 2 Programa de Pós-graduação em Zootecnia - UFV, Viçosa, MG. \\ ${ }^{3}$ Departamento de Zootecnia/UFV, Viçosa, MG. Bolsista do CNPq. \\ ${ }^{4}$ Departamento de Economia Rural/UFV, Viçosa, MG \\ ${ }^{5}$ EMBRAPA Gado de Leite, Juiz de Fora, MG. \\ ${ }^{6}$ Curso de Medicina Veterinária/UNIPAC, Juiz de Fora, MG.
}

RESUMO - Objetivou-se desenvolver uma metodologia alternativa para avaliação bioeconômica de estratégias de alimentação para rebanhos leiteiros e avaliar a produtividade física e a eficiência bioeconômica de sistemas de alimentação para vacas em lactação quando utilizadas diversas estratégias de alimentação à base de volumosos para vacas de cinco níveis de produção de leite. Utilizou-se uma plataforma computacional desenvolvida com os programas CNCPS v.5.0 e planilhas eletrônicas do Microsoft Excell ${ }^{\circledR}$, de forma a simular a produção e as exigências de nutrientes ao longo da lactação de uma vaca com cinco níveis de produção de leite. Utilizaram-se a metodologia alternativa "Ajuste para o Nível de Capital”, foram observados diversos níveis de utilização de concentrados e capital gasto com alimentação por unidade de área e estimadas equações de regressão da produtividade (PROD/ha) e receita menos os custos com alimentação (LUCR/ha) por hectare, em função destes níveis. Esta metodologia não permitiu que ocorresse utilização de diferentes níveis de insumos e de capital por unidade de área, mesmo quando são avaliadas diferentes estratégias de alimentação. Os resultados obtidos comprovaram diferenças nas avaliações bioeconômicas realizadas pela metodologia “Ajuste para o Nível de Capital” em relação à “Tradicional”. De modo geral, principalmente nos maiores níveis de utilização de capital por área, as estratégias baseadas em forragens de melhor qualidade apresentaram resultados superiores na PROD/ha e LUCR/ha. No entanto, nos níveis de menor utilização de capital por área, as estratégias baseadas em forrageiras de alta produtividade por hectare apresentaram melhores resultados nas variáveis analisadas.

Palavras-chave: bovinos de leite, eficiência bioeconômica, simulação

\section{Bioeconomic evaluation of feeding strategies in milk production systems. 2. Alternative methodology: level of capital utilization}

\begin{abstract}
This work was carried out to development a alternative methodology for the bioeconomic evaluation of feeding strategies for dairy herds and evaluate the physical productivity and the bioeconomic efficiency of feeding systems for dairy cows when different feeding strategies forages based are used for cows of five levels of milk yield. The computational platform was developed with the programs CNCPS v5.0 and electronic spreadsheets of Microsoft Excell®, in way to simulate the production and demands of nutrients of a complete lactation for cows of different milk yield levels. Using the alternative methodology "Adjusts for the Level of Capital”, were evaluated different levels of concentrate and capital expense with feeding per area unit and estimate regression equations of productivity (PROD/ha) and income over feed costs (RMCA/ha0 per hectare, in function of these levels. This methodology did not permit the occurrance of the use the different levels of input and capital per unit of area, specifically when are evaluated different feeding strategies. The obtained results showed differences in the bioeconomics evaluations carry out by the "Adjusts for the Level of Capital” methodology in relation to "Traditional" methodology. In general, mainly in the highest levels of use of capital per area, the feeding strategies based on forages of better quality showed the best results in PROD/ha and RMCA/ha. However, in the levels of smaller levels of use of capital per area, the feeding strategies based on forages of high productivity per hectare showed the better results in analyzed variables.
\end{abstract}

Key Words: bioeconomic efficiency, dairy cattle, simulation

Este artigo foi recebido em 1/2/2006 e aprovado em 10/10/2007.

Correspondências devem ser enviadas para francisco.renno@usp.br.

* Endereço atual: Departamento de Nutrição e Produção Animal, Faculdade de Medicina Veterinária e Zootecnia da USP, Campus de Pirassununga, SP. 


\section{Introdução}

A quantificação da produtividade e eficiência bioeconômica de sistemas de alimentação para bovinos, variando o nível de produção e os componentes da alimentação, permitem a definição e a especialização do sistema de produção de leite de acordo com a disponibilidade de recursos naturais, características da base física do sistema de produção e da disponibilidade de alimentos. O estudo do potencial de produção de leite a partir das forrageiras comumente utilizadas nos sistemas de produção adotados no Brasil pode auxiliar na escolha do sistema que será adotado, relacionando-se também com o potencial produtivo dos animais que compõe determinado sistema.

A escolha da melhor estratégia de alimentação, baseada na seleção adequada das forrageiras a serem utilizadas, é influenciada por diversos fatores. Ely (1992) demonstrou que o valor bioeconômico das forragens não é estático, pois depende do nível de produção dos animais, da disponibilidade de alimentos e de seus preços. Desta forma, no momento da escolha do volumoso, fatores como potencial de produção dos animais, disponibilidade de concentrados, disponibilidade da área para produção, aptidão regional da cultura e aptidão do produtor são os aspectos principais a serem considerados (Nogueira, 2004b).

A avaliação bioeconômica de alimentos utilizados em sistemas de produção de leite tem se tornado cada vez mais importante, especialmente pela grande participação dos custos de alimentação nos custos operacionais totais de produção, para diferentes tipos de sistemas de produção (Bath \& Sosnik, 1992; Nogueira, 2004a). Segundo Pereira (2000) e Ferreira (2002), os custos de alimentação podem representar 50 a $60 \%$ dos custos operacionais de produção em sistemas de produção de leite.

Segundo Assis \& Brockington (1999) e Nussio \& Nussio (2003), a escolha da base forrageira em sistemas de produção de leite se caracteriza por ser função multidisciplinar e envolver diferentes setores do sistema de produção, situação em que a técnica de simulação tem se tornado uma ferramenta de grande valia para prever o retorno econômico esperado, especialmente quando avaliadas diferentes fontes de forragem suplementar e em cenários alternativos de produção.

Os estudos que avaliaram o potencial de utilização de diferentes fontes forrageiras em sistemas de produção de leite simularam dietas a partir de um sistema de formulação de rações, como os estudos de Undersander et al. (1993) e Nussio et al. (2001), que utilizaram o NRC (1988) e Nussio \& Nussio (2003), que utilizaram o NRC (2001).
Esses autores avaliaram o potencial de produção de diferentes forrageiras considerando a obtenção de receita menos os custos com alimentação (RMCA) por animal e por unidade de área. Em ambos os estudos, a metodologia considera como restrição nas avaliações a quantidade de terra ou unidades de área disponíveis. Assim, as avaliações por animal não apresentam restrições, enquanto as avaliações por área utilizam somente uma restrição, a disponibilidade de área ou por hectare.

Se o objetivo é a comparação da produção de leite e da geração de receita por unidade de área, a condição básica para a exploração da melhor estratégia de alimentação deve ser baseada no mesmo nível de utilização de capital gasto com alimentação por unidade de área, já que podem existir outras restrições de ordem técnica e econômica que favorecem ou desfavorecem a avaliação de determinada estratégia de alimentação ou base forrageira, comprometendo as análises.

Dessa forma, o objetivo neste estudo foi estabelecer um método de análise da produtividade física e eficiência bioeconômica de alimentos volumosos, considerando restrições múltiplas, para a avaliação de diferentes estratégias de alimentação em sistemas de produção de leite.

\section{Material e Métodos}

Utilizou-se uma plataforma computacional que representa a produção de leite de uma lactação completa desenvolvida utilizando-se o software CNCPS (Cornell Net Carbohydrate and Protein System), versão 5.0 (Fox et al., 2003), para a formulação das dietas. Com estes resultados, por meio de planilhas eletrônicas e utilizando-se o software Microsoft EXCELL ${ }^{\circledR}$, foram realizados os cálculos de eficiência bioeconômica dos diferentes sistemas de alimentação avaliados.

A plataforma computacional utilizada considera as necessidades nutricionais de uma vaca leiteira durante o período de uma lactação, combinado ao valor nutritivo e características agronômicas das forrageiras. Foram simulados cinco níveis de produção de leite por lactação. Modificações nas necessidades dietéticas das vacas decorrentes do aumento de produção ou modificação da base forrageira resultam em alterações no nível de utilização de forragens e de concentrado, alterando a produtividade e a eficiência bioeconômica das estratégias de alimentação. A descrição detalhada da plataforma computacional utilizada foi descrita por Rennó et al. (2008).

Foi utilizado o software CNCPS versão 5.0 (Fox et al., 2003) para o cálculo das dietas e a estimativa das exigências nutricionais e do valor nutritivo dos alimentos. Na estima- 
tiva do valor nutritivo dos alimentos, foram utilizadas as equações do Nível 1. Foi exigido que todas as dietas formuladas atendessem as recomendações de balanço de nutrientes em relação à fase da lactação, independentemente do volumoso ou dos componentes dos concentrados utilizados.

Para a avaliação dos volumosos, foram desenvolvidas sete estratégias de alimentação ao longo da lactação, variando de um sistema de alimentação que utiliza um único volumoso ao longo do ano, a sistemas de alimentação com utilização de pastagens na época das águas (outubro a março) como volumoso exclusivo, e suplementação com silagem ou cana-de-açúcar na época da seca (abril a setembro): Estratégia (EST) 1 - silagem de milho (SIM) como volumoso exclusivo ao longo de toda lactação (SIM); EST 2 - SIM durante a época seca e pastejo em capim-braquiária (BRI) durante a época das águas (SIM+BRI); EST 3 - SIM durante a época seca e pastejo de capim-elefante (NAP) durante a época das águas (SIM+NAP); EST 4 - SIM durante a época seca e pastejo em capim-tifton 85 (TIF) durante a época das águas (SIM+TIF); EST 5 - cana-deaçúcar (CAN) durante a época seca e pastejo de BRI durante a época das águas (CAN+BRI); EST 6 - CAN durante a época seca e pastejo de NAP durante a época das águas (CAN+NAP); EST 7 - CAN durante a época seca e pastejo de TIF durante a época das águas (CAN+TIF).

As estratégias de alimentação foram avaliadas utilizando-se uma metodologia alternativa denominada “Ajuste para o Nível de Capital” (ANUC), baseada na produção por unidade de área, considerando diferentes níveis de utilização de insumos (Input) e gastos com alimentação por hectare. Nesta metodologia primeiramente é corrigido o uso desproporcional de insumos por unidade de área quando são comparadas forrageiras de diferentes qualidades nutricionais e produtividades agrícolas. O uso desproporcional de insumos, quando são comparadas estratégias de alimentação na metodologia tradicional (Tabela 1), é o principal aspecto que deve ser alterado nas avaliações.

Na metodologia ANUC são consideradas duas variáveis limitantes em cada análise: área e nível de Input, representado pelos gastos com concentrados, expressos em kg/ha e R \$/ha, ou área e nível de gasto com alimentação, expressos em $\mathrm{R} \$ /$ ha. Esta metodologia de avaliação é diferente da avaliação convencional, denominada "Tradicional”, na qual são utilizadas avaliações de estratégias de alimentação ou de alimentos considerando somente um fator limitante, avaliando a produção ou receita por animal (por vaca) ou por unidade de área (por hectare). Esta metodologia foi utilizada por Undersander et al. (1993),
Nussio \& Nussio (2003) e Rennó et al. (2008). Com esta metodologia "Tradicional” os alimentos são utilizados livremente, sem nenhuma restrição de utilização de insumos ou de capital, especialmente nas avaliações por unidade de área.

Com a metodologia alternativa ANUC, foram avaliadas a produção de leite (PROD/ha) e a geração de receita por unidade de área (LUCR/ha) considerando diferentes níveis de utilização de insumos e de gasto com alimentação por unidade de área. Como insumo mais importante em rebanhos de gado de leite, conforme demonstrado por Ferreira (2002) e Nogueira (2004a), foi escolhido o nível de concentrado utilizado por hectare como Input representativo do nível de utilização de insumos por unidade de área.

A utilização de concentrado foi avaliada em relação à quantidade física, em $\mathrm{kg}$ de concentrado por hectare (COK/ha), e econômica, em reais de concentrado por hectare (COR/ha), representando a quantidade e qualidade de concentrado utilizada. Para a realização de uma avaliação mais completa, foi utilizado o nível de gasto com alimentação por unidade de área, somando os custos de volumosos e concentrados. A fixação dos níveis de concentrados ou gastos com alimentação por unidade de área, utilizada na metodologia ANUC, possibilita avaliações de diferentes estratégias de alimentação, e de alimentos, com a utilização de restrições de utilização, diferentemente do que ocorre na metodologia tradicional de avaliação.

Para a metodologia ANUC, foram utilizados os dados gerados por meio de simulações, conforme relatado em artigo anterior (Rennó et al., 2008). Em cada estratégia de alimentação avaliada, foi estimada uma equação de regressão com os dados gerados de PROD/ha e LUCR/ha em função da utilização de concentrados por hectare (COK/ha); em função do gasto, em reais, com concentrados por hectare (COR/ha); e do gasto, em reais, com alimentação por hectare (CAL/ha).

Desta forma, foram fixados níveis de gasto com concentrados e alimentação por unidade de área de acordo com os limites máximos de gastos nestas variáveis obtidas nas simulações (Tabelas 1 e 2). Na avaliação da utilização de concentrados (kg/ha), foram fixados os níveis 3.000, 6.000, $9.000,12.000$ e $15.000 \mathrm{~kg} / \mathrm{ha} / \mathrm{ano}$. Na avaliação do gasto com concentrados ( $\mathrm{R} \$ / \mathrm{ha}$ ), foram fixados os níveis 2.500, 5.000, 7.500 e $9.000 \mathrm{R} \$ /$ ha/ano. Na avaliação do gasto com alimentação (R\$/ha), foram fixados os níveis 4.000, 6.000, 8.000 e $10.000 \mathrm{R} \$$ /ha/ano.

As taxas de lotação são diferentes entre as estratégias de alimentação, em virtude das diferenças de produtividade das forrageiras avaliadas e do nível de inclusão de volumosos nas dietas formuladas. Como na obtenção destes dados 
Tabela 1 - Utilização de concentrado, expresso em $\mathrm{kg}(\mathrm{COK} / \mathrm{ha})$ e reais (COK/ha) por hectare, gastos com alimentação por hectare $(\mathrm{R} \$ /$ ha), e relativização desses gastos (entre parênteses e na coluna) em relação a estratégia SIM, em função de cada estratégia de alimentação utilizada e nível de produção



gerados por meio da metodologia tradicional a produção por vaca em cada nível de produção é definida no início das simulações, o resultado são diferenças na quantidade de concentrados e gastos com alimentação por unidade de área.

Na metodologia ANUC, são limitados os gastos com concentrados e alimentação por área, descritos anteriormente, o que, somado ao potencial de produção de leite com a utilização dos volumosos que compõe as estratégias de alimentação, que apresentam valor nutritivo característico, resultam em vacas de níveis de produção distintos para cada nível de limitação de gastos com concentrados e alimentação, e para cada estratégia de alimentação.

Para se obter a produção de leite média por vaca, também foram estimadas equações de regressão da produção de leite por vaca em função da quantidade de concentrado fornecida por animal, em kg de concentrado por vaca (COK/vaca) e do gasto com concentrado em reais por vaca (COR/vaca), e em função do gasto com alimentação (R\$/vaca), para cada estratégia de alimentação. Os dados utilizados foram gerados em estudo anterior (Rennó et al., 2008).

Assim, dividindo o nível de concentrado utilizado por hectare, restringido para valores fixos conforme relatado anteriormente, pela taxa de lotação média estimada para cada estratégia de alimentação, é obtida a quantidade de concentrado disponível por vaca para cada nível de gasto com concentrados e alimentação por unidade de área. Com a utilização das equações de regressão, que estimam a produção de leite por vaca em função do nível de gasto com concentrados e alimentação, foi possível determinar a produção esperada por vaca nas diferentes estratégias.

Com este procedimento foi possível verificar diferenças no nível de produção de leite por vaca quando são comparadas estratégias de alimentação e utilizadas res- 
Tabela 2 - Equações de regressão da produção de leite (PROD/ha), da receita menos os custos de alimentação (LUCR/ha) e da estimativa de produção de leite por animal ( $\mathrm{kg} / \mathrm{vaca} / \mathrm{ano})$, em função do nível de utilização de concentrado $\left(\mathrm{kg} / \mathrm{ha} / \mathrm{ano}^{1} \mathrm{e} \mathrm{R} \$ / \mathrm{ha} / \mathrm{ano}{ }^{2}\right)$, para cada estratégia de alimentação

\begin{tabular}{|c|c|c|c|c|}
\hline Estratégia de alimentação & Equação de regressão ${ }^{1,3}$ & $\mathrm{R}^{2}$ & Equação de regressão ${ }^{2,3}$ & $\mathrm{R}^{2}$ \\
\hline \multicolumn{5}{|c|}{ Produção de leite (PROD/ha) } \\
\hline SIM & $5126,59884+2,69734 * \mathrm{X}$ & 98,40 & $3082,17226+5,28337 * \mathrm{Y}$ & 97,58 \\
\hline SIM+BRI & $5590,80901+2,21019 * \mathrm{X}$ & 99,50 & $3562,12734+4,55076 * \mathrm{Y}$ & 99,79 \\
\hline $\mathrm{SIM}+\mathrm{NAP}$ & $7539,30962+2,14090 * \mathrm{X}$ & 99,41 & $6846,38776+4,44527 * \mathrm{Y}$ & 99,77 \\
\hline SIM+TIF & $5777,64119+2,14723 * X$ & 99,64 & $5518,00482+4,38485 * \mathrm{Y}$ & 99,82 \\
\hline $\mathrm{CAN}+\mathrm{BRI}$ & $4903,01200+2,11432 * X$ & 99,83 & $3551,00093+3,85476 * \mathrm{Y}$ & 99,99 \\
\hline $\mathrm{CAN}+\mathrm{NAP}$ & $7887,53227+2,04508 * \mathrm{X}$ & 99,51 & $7833,25904+3,80205 * \mathrm{Y}$ & 99,97 \\
\hline $\mathrm{CAN}+\mathrm{TIF}$ & $4735,40562+2,07916 * X$ & 99,82 & $5824,20533+3,65034 * \mathrm{Y}$ & 99,96 \\
\hline \multicolumn{5}{|c|}{ Receita (LUCR/ha) } \\
\hline SIM & $-160,80520+0,79856 * \mathrm{X}$ & 95,23 & $-747,48437+1,55972 * \mathrm{Y}$ & 93,70 \\
\hline $\mathrm{SIM}+\mathrm{BRI}$ & $1000,45627+0,56168 * \mathrm{X}$ & 98,38 & $480,49090+1,15754 * \mathrm{Y}$ & 98,54 \\
\hline SIM+NAP & $1690,92287+0,54335 * X$ & 98,26 & $1511,97116+1,12880 * \mathrm{Y}$ & 98,78 \\
\hline SIM+TIF & $1368,34433+0,52862 * \mathrm{X}$ & 98,08 & $1301,43558+1,08030 * \mathrm{Y}$ & 98,47 \\
\hline $\mathrm{CAN}+\mathrm{BRI}$ & $989,25265+0,46582 * \mathrm{X}$ & 99,34 & $686,21582+0,84996 * \mathrm{Y}$ & 99,75 \\
\hline $\mathrm{CAN}+\mathrm{NAP}$ & $2129,94397+0,44511 * \mathrm{X}$ & 98,93 & $2113,47455+0,82797 * \mathrm{Y}$ & 99,55 \\
\hline CAN+TIF & $1400,27734+0,42611 * X$ & 98,64 & $1620,61952+0,74853 * \mathrm{Y}$ & 98,94 \\
\hline
\end{tabular}

Produção de leite $(\mathrm{kg} / \mathrm{vaca} / \mathrm{ano})$

$\begin{array}{lcccc}\text { SIM } & 1195,77166+2,72904 *(\mathrm{X}) / 4,14 & 97,51 & 677,62738+5,36597 *(\mathrm{Y}) / 4,14 & 97,01 \\ \text { SIM+BRI } & 1583,86058+2,07887 *(\mathrm{X}) / 4,18 & 99,05 & 1028,35983+4,37790 *(\mathrm{Y}) / 4,18 & 99,58 \\ \text { SIM+NAP } & 1585,91476+2,03388 *(\mathrm{X}) / 5,45 & 99,06 & 1440,67886+4,24926 *(\mathrm{Y}) / 5,45 & 99,54 \\ \text { SIM+TIF } & 1747,50633+1,98600 *(\mathrm{X}) / 4,00 & 99,14 & 1670,87428+4,07325 *(\mathrm{Y}) / 4,00 & 99,47 \\ \text { CAN+BRI } & 1097,08071+1,99684 *(\mathrm{X}) / 5,86 & 99,57 & 807,01605+3,69686 *(\mathrm{Y}) / 5,86 & 99,96 \\ \text { CAN+NAP } & 1230,56242+1,90716 *(\mathrm{X}) / 8,58 & 99,06 & 1200,36141+3,56523 *(\mathrm{Y}) / 8,58 & 99,89 \\ \text { CAN+TIF } & 1136,05727+1,95395 *(\mathrm{X}) / 5,55 & 99,45 & 1364,21982+3,39466 *(\mathrm{Y}) / 5,55 & 99,74\end{array}$

${ }^{1} \mathrm{X}=\mathrm{kg}$ conc. $/ \mathrm{ha} ;{ }^{2} \mathrm{Y}=\mathrm{R} \$$ conc./ha; ${ }^{3}$ Todas as equações $\mathrm{P}<0,01$.

$\mathrm{SIM}=$ silagem de milho como volumoso exclusivo; SIM+BRI = silagem de milho durante a época seca e pastejo em capim-braquiária durante a época das águas; SIM+NAP = silagem de milho durante a época seca e pastejo de capim-elefante durante a época das águas; SIM+TIF = silagem de milho durante a época seca e pastejo em capim-tifton 85 durante a época das águas; CAN+BRI = cana-de-açúcar durante a época seca e pastejo em capim-braquiária durante a época das águas; CAN+NAP = cana-de-açúcar durante a época seca e pastejo de capim-elefante durante a época das águas; CAN+TIF = cana-de-açúcar durante a época seca e pastejo em capim-tifton 85 durante a época das águas.

trições múltiplas, unidade de área e nível de Input, na metodologia ANUC. Para a estimativa das equações de regressão, foi utilizado o software SAS (Statistical Analysis System), utilizando-se o procedimento PROC REG (SAS, 1999).

\section{Resultados e Discussão}

Foram observadas diferenças na utilização de concentrados e nos gastos com alimentação por unidade de área em mesmo nível de produção por animal quando utilizada a metodologia "Tradicional” de avaliação (Tabela 1). O gasto com concentrado por hectare, em $\mathrm{kg}$ (COK/ha) e em reais (COR/ha), no mesmo nível de produção, foi significativamente relacionado à qualidade das forrageiras utilizadas nas estratégias de alimentação (Tabela 1). As estratégias baseadas na utilização da silagem de milho como volumoso exclusivo (SIM) ou em associação com pastagens (SIM+BRI, SIM+NAP e SIM+TIF) resultaram na menor quantidade de concentrados utilizados, nas duas formas de avaliação, quando comparado às estratégias que utilizaram cana-de-açúcar e pastagens (CAN+BRI, $\mathrm{CAN}+\mathrm{NAP}$ e $\mathrm{CAN}+\mathrm{TIF}$ ).

Entre as combinações com pastagens, utilizando silagem de milho ou cana-de-açúcar, a utilização do capim-tifton 85 (TIF), de melhor qualidade (Tedeschi et al., 2002; Valadares Filho et al., 2003), resultou em menor utilização de concentrado e de capital por hectare, quando comparado ao capimelefante (NAP) e ao capim-braquiária (BRI). Entre esses dois últimos, a utilização do capim-braquiária resultou em menor utilização de concentrado por hectare em relação ao capim-elefante.

No nível baixo de produção, a estratégia SIM apresentou maior gasto relativo de concentrados. No entanto, nos demais níveis, foram observados níveis de utilização de concentrado por hectare até 364,13\% maiores que os utilizados na estratégia SIM. Desta forma, nas estratégias em que foram utilizadas pastagens e cana-de-açúcar, gastou-se muito mais Input (concentrados) por hectare do que na estratégia baseada em silagem de milho como volumoso exclusivo (SIM).

De forma semelhante, avaliando os gastos com alimentação por unidade de área nas estratégias baseadas em 
silagem de milho, exclusivamente ou em combinação com pastagens (SIM, SIM+BRI, SIM+NAP e SIM+TIF), despendeu-se relativamente menor quantidade de gastos com alimentação por hectare que nas estratégias baseadas em cana-de-açúcar e pastagens (CAN+BRI, CAN+NAP e CAN+TIF). Exceção para a estratégia SIM no nível baixo de produção, que novamente apresentou maior gasto com alimentação (Tabela 1).

Apesar de os gastos com alimentação por unidade de área serem relativamente mais próximos da estratégia de alimentação referência (SIM) que a utilização de concentrado por unidade de área, na mesma comparação, as diferenças nos gastos entre as estratégias ainda são grandes. Foram observados níveis de gasto com alimentação por hectare até 274,95\% superiores ao da estratégia SIM.

A metodologia "Tradicional” resulta em níveis de utilização de insumos e de gastos com alimentação diferentes entre as estratégias de alimentação (Tabela 1). Assim, nas avaliações por unidade de área estão sendo comparadas estratégias de alimentação sem restrição alguma do uso de concentrados ou de gastos com alimentação quando estas estratégias são comparadas.

Nesse caso, estratégias de alimentação que resultam em maiores níveis de utilização de concentrados e gastos com alimentação por unidade de área são amplamente favorecidas, resultando artificialmente em maior PROD/ha e LUCR/ha. No entanto, estes resultados ocorreram em razão do maior gasto com concentrados e alimentação, e não de alguma vantagem diretamente relacionada à estratégia de alimentação, levando a conclusões equivocadas relacionadas à produtividade e rentabilidade de diferentes estratégias de alimentação por unidade de área.

Quanto à silagem de milho, apesar de ser volumoso referência por seu valor nutritivo e sua intensa utilização em sistemas de produção de leite, são comuns argumentos desfavoráveis a sua utilização em função da menor rentabilidade obtida por unidade de área em comparação a outras forrageiras, apesar de esse volumoso propiciar o menor custo de alimentação e a maior rentabilidade por vaca (Nussio et al., 1998, Nussio \& Nussio, 2003).

Segundo os autores citados anteriormente, se o objetivo for maximizar a receita obtida por hectare, esta receita é fortemente influenciada pela capacidade de suporte das forrageiras, em todos os níveis de produção. Mesmo que determinada estratégia apresente menores custos de alimentação e, portanto, maior receita menos custos de alimentação por vaca, a taxa de lotação que essa estratégia pode ser submetida altera marcadamente a receita por área.

Analisando os resultados desse estudo e os apresentados por Nussio et al. (1998) e Nussio \& Nussio (2003), que utilizaram a metodologia de simulação, e os resultados de Costa et al. (2005) e Oliveira et al. (2004), em experimentos com animais, verifica-se que as dietas formuladas com silagem de milho, dentro de um mesmo nível de produção, sempre apresentam maiores proporções de volumoso se comparada a qualquer outra forrageira. Esta condição resulta em menor utilização de concentrados nas dietas, com melhor renda por vaca.

No entanto, considerando a maior utilização desse volumoso por animal e a menor produtividade agrícola por unidade de área do milho em comparação a outras culturas, a utilização da silagem de milho como volumoso resulta em menor capacidade de suporte, e conseqüentemente, como o nível de produção por vaca é fixo, em menor retorno econômico por unidade de área.

Desta forma, é importante saber se a maior proporção de volumoso nas dietas com silagem de milho influencia os resultados econômicos da produção de leite, avaliada por unidade de área, ou se somente a menor produtividade agrícola explica a reduzida capacidade de suporte e o desempenho inferior das estratégias de alimentação baseadas em silagem de milho como volumoso exclusivo, na mesma base de comparação.

Nas avaliações por animal, o melhor resultado depende do menor custo de alimentação, uma vez que a receita obtida é fixa para determinado nível de produção em todas as estratégias de alimentação. Nas avaliações por unidade de área, o melhor resultado depende da maior produtividade agrícola e conseqüentemente maior capacidade de suporte das forrageiras nas estratégias de alimentação, pois, como a receita obtida é fixa por vaca para determinado nível de produção, quanto maior o número de animais por hectare, maior a receita por área, resultando em maior produtividade obtida por hectare.

Nas avaliações por unidade de área na metodologia “Tradicional”, a utilização de concentrados por hectare é livre, o que caracteriza diferentes níveis de Input quando avaliamos a mesma área (Tabela 1). Assim, a utilização de diferentes níveis de concentrado e gastos com alimentação por hectare, quando é comparado o mesmo nível de produção, nas variadas estratégias de alimentação, pode estar subestimando ou superestimando a produtividade e renda das estratégias de alimentação.

A silagem de milho poderia ter seu potencial de produção e geração de receita por hectare subestimado, principalmente devido a suas características nutricionais favoráveis, que resultam em maior proporção de utilização deste volumoso nas dietas de vacas leiteiras e, conseqüentemente, diminuem sua capacidade de suporte. De forma semelhante, a avaliação da utilização de cana-de-açúcar poderia estar 
Tabela 3 - Produção de leite (PROD/ha), receita menos os custos de alimentação (LUCR/ha) e estimativa de produção de leite por animal (kg/vaca/ano), em função do nível de utilização de concentrado (kg/ha/ano), para cada estratégia de alimentação

\begin{tabular}{|c|c|c|c|c|c|}
\hline Estratégia de alimentação & \multicolumn{5}{|c|}{ Nível de concentrado (kg/ha/ano) } \\
\hline & \multicolumn{5}{|c|}{ Produção de leite (PROD/ha) } \\
\hline SIM & $13.218,6$ & $21.310,6$ & $29.402,7$ & $37.494,7$ & $45.586,7$ \\
\hline SIM+BRI & $12.221,4$ & $18.851,9$ & $25.482,5$ & $32.113,1$ & $38.743,7$ \\
\hline SIM+NAP & $13.962,0$ & $20.384,7$ & $26.807,4$ & $33.230,1$ & $39.652,8$ \\
\hline $\mathrm{CAN}+\mathrm{NAP}$ & $14.022,8$ & $20.158,0$ & $26.293,3$ & $32.428,5$ & $38.563,7$ \\
\hline \multirow[t]{2}{*}{ CAN+TIF } & $10.972,9$ & $17.210,4$ & $23.447,8$ & $29.685,3$ & $35.922,8$ \\
\hline & \multicolumn{5}{|c|}{ Receita (LUCR/ha) } \\
\hline SIM & $2.234,9$ & $4.630,6$ & $7.026,2$ & $9.421,9$ & $11.817,6$ \\
\hline SIM+BRI & $2.685,5$ & $4.370,5$ & $6.055,6$ & $7.740,6$ & $9.425,7$ \\
\hline \multirow[t]{2}{*}{$\mathrm{CAN}+\mathrm{TIF}$} & $2.678,6$ & $3.956,9$ & $5.235,3$ & $6.513,6$ & $7.791,9$ \\
\hline & \multicolumn{5}{|c|}{ Produção de leite (kg/vaca/ano) } \\
\hline SIM & $3.177,4$ & $5.159,1$ & $7.140,7$ & $9.122,4$ & $11.104,1$ \\
\hline SIM+BRI & $3.086,2$ & $4.588,6$ & $6.091,0$ & $7.593,3$ & $9.095,7$ \\
\hline SIM+NAP & $2.709,8$ & $3.833,7$ & $4.957,6$ & $6.081,5$ & $7.205,4$ \\
\hline SIM+TIF & $3.249,7$ & $4.751,9$ & $6.254,1$ & $7.756,4$ & $9.258,6$ \\
\hline $\mathrm{CAN}+\mathrm{BRI}$ & $2.133,5$ & $3.169,9$ & $4.206,3$ & $5.242,7$ & $6.279,2$ \\
\hline $\mathrm{CAN}+\mathrm{NAP}$ & $1.907,5$ & $2.584,5$ & $3.261,4$ & $3.938,4$ & $4.615,3$ \\
\hline CAN+TIF & $2.209,1$ & $3.282,2$ & $4.355,3$ & $5.428,4$ & $6.501,4$ \\
\hline
\end{tabular}

$\mathrm{SIM}$ = silagem de milho como volumoso exclusivo; SIM+BRI = silagem de milho durante a época seca e pastejo em capim-braquiária durante a época das águas; SIM+NAP = silagem de milho durante a época seca e pastejo de capim-elefante durante a época das águas; SIM+TIF = silagem de milho durante a época seca e pastejo em capim-tifton 85 durante a época das águas; CAN+BRI = cana-de-açúcar durante a época seca e pastejo em capim-braquiária durante a época das águas; CAN+NAP = cana-de-açúcar durante a época seca e pastejo de capim-elefante durante a época das águas; CAN+TIF = cana-de-açúcar durante a época seca e pastejo em capim-tifton 85 durante a época das águas.

superestimada, visto que este volumoso estaria sendo beneficiado nas avaliações por unidade de área simplesmente por apresentar características nutricionais que limitam sua utilização nas dietas de vacas em lactação, resultando na menor proporção deste volumoso nas dietas e, conseqüentemente, auxiliando a manutenção de maior capacidade de suporte.

A responsabilidade da super ou subestimação do potencial de produção dos diferentes volumosos está na fixação do nível de produção por vaca no início das simulações na metodologia “Tradicional”. Quando é fixado o nível de produção, são atendidas as necessidades de nutrientes dos animais neste nível, obtendo-se os custos de alimentação para cada estratégia de alimentação, e, relacionando com a receita da produção de leite, se obtêm a receita menos os custos de alimentação, e a avaliação por animal.

No entanto, quando esta avaliação é realizada por unidade de área, a obtenção da receita por área é multiplicada pela capacidade de suporte de cada estratégia de alimentação, obtendo-se, desta forma, a receita por unidade de área. Nessa situação a utilização de concentrados e gastos com alimentação é livre, sem restrições, permitindo a ocorrência do verificado na Tabela 1.

A avaliação da produtividade e receita por unidade de área, em sistemas bioeconômicos com bovinos, deve considerar a priori das avaliações, o gasto com concentrados e com alimentação, sob pena de ocorrer super ou subestimação do potencial de produtividade das estratégias de alimentação avaliadas.

Com a utilização de concentrado (COK/ha), as estratégias de alimentação SIM, SIM+BRI, SIM+NAP e SIM+TIF apresentaram os melhores resultados de produtividade (PROD/ha) e receita (LUCR/ha) em relação às estratégias com cana-de-açúcar e pastagens (CAN+BRI, CAN+NAP e CAN+TIF) (Tabela 2). Todas as equações apresentaram alto coeficiente de determinação, comprovando bom ajuste em relação aos dados avaliados. Considerando a estratégia SIM como referência, as estratégias que utilizaram a combinação da silagem de milho e cana-de-açúcar com pastagens, apresentaram na média, desempenho de 87,74 e 83,52\% na PROD/ha, e 89,01 e 78,53\% na LUCR/ha, respectivamente, em relação à estratégia SIM (Tabelas 3 e 4). Nos três maiores 
níveis de utilização de concentrados, a estratégia SIM apresentou sempre os melhores resultados, sendo, na média, 13,87 e $17,87 \%$ superior na PROD/ha e 16,29 e $27,25 \%$ superior na LUCR/ha, respectivamente, em relação às estratégias baseadas em silagem de milho e cana-de-açúcar combinadas com pastagens.

No menor nível de utilização de concentrado (3.000 kg/ha), a utilização de silagem de milho e cana-de-açúcar, combinada com pastagens, apresentou melhores resultados em comparação à estratégia SIM (Tabelas 3 e 4). A estratégia SIM apresentou PROD/ha 3,16 e 8,61\% maior que as estratégias à base de silagem de milho e cana-de-açúcar combinadas com pastagens, respectivamente. Entretanto, a geração de receita por hectare (LUCR/ha) foi 30,44\% inferior na estratégia SIM em relação às demais.

Entre as estratégias baseadas em cana-de-açúcar, a estratégia CAN+NAP, por possibilitar alta taxa de lotação, apresentou resultados na PROD/ha e LUCR/ha, nos dois primeiros níveis de utilização de concentrado (3.000 e $6.000 \mathrm{~kg} / \mathrm{ha} / \mathrm{ano})$, próximos aos obtidos com as estratégias com utilização de silagem de milho.
A avaliação do gasto com concentrados em reais (COR/ha) também resultou em maior PROD/ha e LUCR/ha nas estratégias de alimentação que utilizaram silagem de milho. As estratégias SIM+BRI, SIM+NAP e SIM+TIF apresentaram, na média, PROD/ha e LUCR/ha 92,20 e 90,95\%, respectivamente, da observada na estratégia SIM. As estratégias à base de cana-de-açúcar resultaram em PROD/ha e LUCR/ha de somente 81,51 e 73,47\%, respectivamente, da apresentada na estratégia SIM.

No nível de gasto com concentrados de R \$ 5.000/ha, os resultados das estimativas de $\mathrm{PROD} / \mathrm{ha}$ e LUCR/ha foram semelhantes entre as estratégias de alimentação. No menor nível de gasto com concentrados avaliado, R\$ 2.500/ha, a estratégia SIM apresentou resultados inferiores às demais estratégias, apresentando LUCR/ha 23,85 e 10,90\% inferior à utilização de silagem de milho ou cana-de-açúcar combinadas com pastagens, respectivamente. Apesar de a estratégia SIM ter apresentado níveis semelhantes às demais na estimativa da PROD/ha, a receita por unidade de área (LUCR/ha) foi comprometida pelo maior custo de alimentação e foi inferior às demais estratégias na avaliação da receita obtida por área.

Tabela 4 - Produção de leite (PROD/ha), receita menos os custos de alimentação (LUCR/ha) e estimativa de produção de leite por animal (kg/vaca/ano), em função do nível de utilização de concentrado ( $\mathrm{R} \$ / \mathrm{ha} / \mathrm{ano})$, para cada estratégia de alimentação

\begin{tabular}{|c|c|c|c|c|}
\hline \multirow[t]{2}{*}{ Estratégia de alimentação } & \multicolumn{4}{|c|}{ Nível de concentrado (kg/ha/ano) } \\
\hline & 2.500 & 5.000 & 7.500 & 9.000 \\
\hline & \multicolumn{4}{|c|}{ Produção de leite (PROD/ha) } \\
\hline SIM & $16.290,6$ & $29.499,0$ & $42.707,4$ & $50.632,5$ \\
\hline SIM+BRI & 14.939,0 & $26.315,9$ & $37.692,8$ & $44.519,0$ \\
\hline SIM+NAP & $17.959,6$ & $29.072,7$ & $40.185,9$ & $46.853,8$ \\
\hline SIM+TIF & $16.480,1$ & $27.442,3$ & $38.404,4$ & $44.981,7$ \\
\hline $\mathrm{CAN}+\mathrm{BRI}$ & $13.187,9$ & $22.824,8$ & $32.461,7$ & $38.243,8$ \\
\hline $\mathrm{CAN}+\mathrm{NAP}$ & $17.338,4$ & $26.843,5$ & $36.348,6$ & $42.051,7$ \\
\hline $\mathrm{CAN}+\mathrm{TIF}$ & $14.950,1$ & $24.075,9$ & $33.201,8$ & $38.677,3$ \\
\hline
\end{tabular}

\begin{tabular}{|c|c|c|c|c|}
\hline \multirow[b]{2}{*}{ SIM } & \multicolumn{4}{|c|}{ Receita (LUCR/ha) } \\
\hline & $3.151,8$ & $7.051,1$ & $10.950,4$ & $13.290,0$ \\
\hline SIM+BRI & $3.374,3$ & $6.268,2$ & $9.162,0$ & $10.898,4$ \\
\hline $\mathrm{SIM}+\mathrm{NAP}$ & $4.334,0$ & $7.156,0$ & $9.978,0$ & $11.671,2$ \\
\hline SIM+TIF & $4.002,2$ & $6.702,9$ & $9.403,7$ & $11.024,1$ \\
\hline $\mathrm{CAN}+\mathrm{BRI}$ & $2.811,1$ & $4.936,0$ & $7.060,9$ & $8.335,9$ \\
\hline $\mathrm{CAN}+\mathrm{NAP}$ & $4.183,4$ & $6.253,3$ & $8.323,2$ & $9.565,2$ \\
\hline \multirow[t]{2}{*}{$\mathrm{CAN}+\mathrm{TIF}$} & $3.491,9$ & $5.363,3$ & $7.234,6$ & $8.357,4$ \\
\hline & \multicolumn{4}{|c|}{ Produção de leite (kg/vaca•ano) } \\
\hline SIM & $3.924,7$ & $7.171,7$ & $10.418,7$ & $12.366,9$ \\
\hline SIM+BRI & $2.931,9$ & $5.041,1$ & $7.150,4$ & $8.415,9$ \\
\hline SIM+NAP & $2.717,9$ & $4.283,3$ & $5.848,7$ & $6.787,9$ \\
\hline $\mathrm{SIM}+\mathrm{TIF}$ & $3.390,7$ & $5.444,7$ & $7.498,7$ & $8.731,1$ \\
\hline $\mathrm{CAN}+\mathrm{BRI}$ & $1.924,8$ & $3.204,0$ & $4.483,2$ & $5.250,7$ \\
\hline $\mathrm{CAN}+\mathrm{NAP}$ & $1.803,9$ & $2.647,6$ & $3.491,3$ & $3.997,5$ \\
\hline CAN+TIF & $2.334,2$ & $3.577,1$ & $4.819,9$ & $5.565,7$ \\
\hline
\end{tabular}

SIM = silagem de milho como volumoso exclusivo; SIM+BRI= silagem de milho durante a época seca e pastejo em capim-braquiária durante a época das águas; SIM+NAP = silagem de milho durante a época seca e pastejo de capim-elefante durante a época das águas; SIM+TIF = silagem de milho durante a época seca e pastejo em capim-tifton 85 durante a época das águas; CAN+BRI = cana-de-açúcar durante a época seca e pastejo em capim-braquiária durante a época das águas; CAN+NAP = cana-de-açúcar durante a época seca e pastejo de capim-elefante durante a época das águas; CAN+TIF = cana-de-açúcar durante a época seca e pastejo em capim-tifton 85 durante a época das águas. 
Nas Tabelas 3 e 4, também estão descritas as estimativas de produção de leite por vaca/lactação nos níveis de concentrado avaliados. Como as forrageiras apresentam diferentes valores nutritivos e permitem diferentes taxas de lotação, restringindo a utilização de concentrados por área, e, conseqüentemente, por animal, o nível de produção por vaca é proporcional à combinação do valor nutritivo das forragens e da quantidade disponível de concentrados. Utilizando as equações de regressão descritas na Tabela 2, foi possível determinar a produção/vaca/ano de cada estratégia de alimentação nos níveis de utilização dos concentrados avaliados (Tabelas 3 e 4).

Considerando um nível fixo de gasto com concentrados por área, na metodologia ANUC, as produções por vaca não serão mais iguais nas estratégias de alimentação. $\mathrm{Na}$ avaliação da PL/vaca/ano, considerando diferentes COK/ha (Tabela 3), as estratégias à base de silagem de milho e cana-de-açúcar com pastagens apresentaram, na média, PL/vaca/ano de 80,77 a 55,19\% da observada na estratégia SIM para o mesmo nível de utilização de concentrados. Considerando a avaliação do gasto com concentrados em COR/ha (Tabela 4), a estratégia SIM resultou em produção de leite por vaca, na média, cerca de 32,86 e $57,60 \%$ superiores às estratégias à basea de silagem de milho e cana-de-açúcar e pastagens, respectivamente, para mesmo nível de gasto com concentrados.

Todas as equações utilizadas para estimar a PROD/ha e a LUCR/ha em relação ao nível de gasto com alimentação por unidade de área (CAL/ha) apresentaram alto coeficiente de determinação, demonstrando bom ajuste em relação aos dados avaliados (Tabela 5).

A PROD/ha e a LUCR/ha da estratégia SIM foi, na média, 2,96 e 10,28\%, e 5,72 e 19,86\% superiores às estratégias que combinaram silagem de milho(SIM+BRI, SIM+NAP e SIM+TIF) e cana-de-açúcar (CAN+BRI, CAN+NAP e CAN+TIF) com pastagens, respectivamente (Tabela 6). Considerando os dois maiores níveis de gastos com alimentação por hectare (R \$ 8.000 e R \$10.000/ha), a estratégia SIM apresentou PROD/ha e LUCR/ha 11,76 e 19,63\% superior à média das estratégias SIM+BRI, SIM+NAP e SIM+TIF, respectivamente, e 20,81 e 34,79\% superior à média das estratégias CAN+BRI, CAN+NAP e CAN+TIF, respectivamente. Nesses dois níveis de utilização de capital com alimentação por unidade de área, a utilização da estratégia SIM claramente apresentou resultados superiores aos das demais estratégias de alimentação (Tabela 6).

No nível intermediário de gastos com alimentação (R \$6.000/ha), as estratégias SIM+BRI, SIM+NAP e SIM+TIF foram em média 4,98 e 10,49\% superiores na PROD/ha e LUCR/ha, respectivamente, em relação à estratégia SIM.
Tabela 5 - Equações de regressão da produção de leite (PROD/ ha), da receita menos os custos de alimentação (LUCR/ha), e da estimativa de produção de leite por animal ( $\mathrm{kg} / \mathrm{vaca} / \mathrm{ano})$ em função do nível de utilização de capital ( $\mathrm{R} \$ /$ ha/ano), para cada estratégia de alimentação

\begin{tabular}{|c|c|c|}
\hline $\begin{array}{l}\text { Estratégia de } \\
\text { alimentação }\end{array}$ & $\begin{array}{l}\text { Equação de } \mathrm{de}^{1,2} \\
\text { regressão }\end{array}$ & $\mathrm{R}^{2}$ \\
\hline & Produção de leite (PROD/ha) & \\
\hline SIM & $-14530,00000+6,35451 * X$ & 98,14 \\
\hline $\mathrm{SIM}+\mathrm{BRI}$ & $-1829,80625+4,34764 * X$ & 99,58 \\
\hline $\mathrm{SIM}+\mathrm{NAP}$ & $-937,98430+4,33684 * X$ & 99,47 \\
\hline $\mathrm{SIM}+\mathrm{TIF}$ & $-187,66079+4,19454 * X$ & 99,27 \\
\hline CAN+BRI & $-344,79729+3,79003 * X$ & 99,87 \\
\hline $\mathrm{CAN}+\mathrm{NAP}$ & $1567,52143+3,75072 * X$ & 99,82 \\
\hline \multirow[t]{2}{*}{ CAN+TIF } & $1578,15161+3,57657 * X$ & 99,62 \\
\hline & Receita (LUCR/ha) & \\
\hline SIM & $-7034,69686+2,07660 * X$ & 95,97 \\
\hline $\mathrm{SIM}+\mathrm{BRI}$ & $-886,02671+1,10499 * X$ & 98,49 \\
\hline $\mathrm{SIM}+\mathrm{NAP}$ & $-454,16444+1,09976 * X$ & 98,07 \\
\hline SIM+TIF & $-90,95221+1,03088 * X$ & 97,20 \\
\hline CAN+BRI & $-167,06837+0,83502 * x$ & 99,40 \\
\hline CAN+NAP & $758,89006+0,81598 * X$ & 99,10 \\
\hline \multirow[t]{2}{*}{ CAN+TIF } & $764,08231+0,73166 * X$ & 97,90 \\
\hline & Produção de leite (kg/vaca•ano) & \\
\hline SIM & $-1875,35239+5,10254 *(X) / 4,14$ & 98,11 \\
\hline $\mathrm{SIM}+\mathrm{BRI}$ & $-575,10798+4,45022 *(X) / 4,18$ & 99,45 \\
\hline $\mathrm{SIM}+\mathrm{NAP}$ & $-222,59897+4,37744 *(X) / 5,45$ & 99,27 \\
\hline $\mathrm{SIM}+\mathrm{TIF}$ & $-84,11964+4,22437 *(\mathrm{X}) / 4,00$ & 98,61 \\
\hline $\mathrm{CAN}+\mathrm{BRI}$ & $-100,04265+3,81872 *(X) / 5,86$ & 99,72 \\
\hline $\mathrm{CAN}+\mathrm{NAP}$ & $234,75206+3,71446 *(X) / 8,58$ & 99,61 \\
\hline $\mathrm{CAN}+\mathrm{TIF}$ & $363,09251+3,52486 *(X) / 5,55$ & 98,94 \\
\hline
\end{tabular}

$1 \mathrm{X}=\mathrm{R} \$$ cap $/$ ha; 2 Todas as equações $\mathrm{P}<0,01$.

$\mathrm{SIM}=$ silagem de milho como volumoso exclusivo; $\mathrm{SIM}+\mathrm{BRI}=$ silagem de milho durante a época seca e pastejo em capim-braquiária durante a época das águas; SIM+NAP = silagem de milho durante a época seca e pastejo de capim-elefante durante a época das águas; SIM+TIF = silagem de milho durante a época seca e pastejo em capim-tifton 85 durante a época das águas; $\mathrm{CAN}+\mathrm{BRI}=$ cana-de-açúcar durante a época seca e pastejo em capim-braquiária durante a época das águas; $\mathrm{CAN}+\mathrm{NAP}=$ cana-de-açúca durante a época seca e pastejo de capim-elefante durante a época das águas; CAN+TIF = cana-de-açúcar durante a época seca e pastejo em capim-tifton 85 durante a época das águas.

Nesse nível de gasto com alimentação por hectare, a estratégia SIM apresentou resultados semelhantes às estratégias CAN+BRI, CAN+NAP e CAN+TIF. No menor nível de gasto com alimentação por unidade de área, a estratégia SIM apresentou resultados modestos. Na média, avaliando em conjunto as estratégias SIM+BRI, SIM+NAP, SIM+TIF, CAN+BRI, CAN+NAP e CAN+TIF, a PROD/ha e a LUCR/ha foram 46,69 e 193,54\% superiores, respectivamente, à estratégia SIM (Tabela 6).

A utilização da metodologia ANUC na avaliação econômica de volumosos modifica os resultados observados na avaliação bioeconômica de estratégias de alimentação quando considerada a metodologia "Tradicional” de avaliação (Rennó et al., 2008), que tende a superestimar o 
Tabela 6 - Produção de leite (PROD/ha), receita menos os custos de alimentação (LUCR/ha) e estimativa de produção de leite por animal ( $\mathrm{kg} / \mathrm{vaca} / \mathrm{ano})$, em função do nível de utilização de capital gasto com alimentação por hectare (R\$/ha/ano), para cada estratégia de alimentação

\begin{tabular}{lccc}
\hline Estratégia de alimentação & \multicolumn{3}{c}{ Nível de capital (R\$/ha/ano) } \\
\cline { 2 - 4 } & 4.000 & 6.000 & 8.000 \\
\hline & & Produção de leite (PROD/ha) & 10.000 \\
SIM & $10.888,0$ & $23.597,1$ & $36.306,1$ \\
SIM+BRI & $15.560,8$ & $24.256,0$ & $32.951,3$ \\
SIM+NAP & $16.409,4$ & $25.083,1$ & $33.756,7$ \\
SIM+TIF & $16.590,5$ & $24.979,6$ & $33.368,7$ \\
CAN+BRI & $14.815,3$ & $22.395,4$ & $29.975,4$ \\
CAN+NAP & $16.570,4$ & $24.071,8$ & $31.573,3$ \\
CAN+TIF & $15.884,4$ & $23.037,6$ & $30.190,7$
\end{tabular}

Receita (LUCR/ha)

\begin{tabular}{|c|c|c|c|c|}
\hline SIM & $1.271,7$ & $5.424,9$ & $9.578,1$ & $13.731,3$ \\
\hline SIM+BRI & $3.533,9$ & $5.743,9$ & $7.953,9$ & $10.163,9$ \\
\hline SIM+NAP & $3.944,9$ & $6.144,4$ & $8.343,9$ & $10.543,4$ \\
\hline SIM+TIF & $4.032,6$ & $6.094,3$ & $8.156,1$ & $10.217,8$ \\
\hline $\mathrm{CAN}+\mathrm{BRI}$ & $3.173,0$ & $4.843,1$ & $6.513,1$ & $8.183,1$ \\
\hline $\mathrm{CAN}+\mathrm{NAP}$ & $4.022,8$ & $5.654,8$ & $7.286,7$ & $8.918,7$ \\
\hline CAN+TIF & $3.690,7$ & $5.154,0$ & $6.617,4$ & $8.080,7$ \\
\hline
\end{tabular}

Produção de leite (kg/vaca/ano)

$\begin{array}{lrrrr}\text { SIM } & 2.659,6 & 5.764,1 & 8.868,6 & 11.973,0 \\ \text { SIM+BRI } & 3.748,5 & 5.843,1 & 7.937,8 & 8.920,8 \\ \text { SIM+NAP } & 3.022,5 & 4.620,2 & 6.217,8 & 7.815,5 \\ \text { SIM+TIF } & 4.183,0 & 6.298,2 & 8.413,4 & 10.528,5 \\ \text { CAN+BRI } & 2.563,2 & 3.874,6 & 5.186,0 & 6.497,5 \\ \text { CAN+NAP } & 1.960,6 & 2.848,1 & 3.735,7 & 4.623,2 \\ \text { CAN+TIF } & 2.907,8 & 4.217,3 & 5.526,7 & 6.836,2\end{array}$

SIM = silagem de milho como volumoso exclusivo; SIM+BRI = silagem de milho durante a época seca e pastejo em capim-braquiária durante a época das águas; SIM+NAP = silagem de milho durante a época seca e pastejo de capim-elefante durante a época das águas; SIM+TIF = silagem de milho durante a época seca e pastejo em capim-tifton 85 durante a época das águas; CAN+BRI = cana-de-açúcar durante a época seca e pastejo em capim-braquiária durante a época das águas; CAN+NAP = cana-de-açúcar durante a época seca e pastejo de capim-elefante durante a época das águas; CAN+TIF = cana-de-açúcar durante a época seca e pastejo em capim-tifton 85 durante a época das águas.

potencial de geração de receita das estratégias de alimentação quando utilizados volumosos de alta produtividade agrícola por unidade de área.

A combinação da alta produtividade agrícola, somada às características nutritivas que limitam a utilização destes volumosos nas dietas (como exemplo a cana-de-açúcar), permite que seja obtida maior taxa de lotação para essas forrageiras. Como não é considerada nenhuma restrição sobre a utilização de concentrados e gastos com alimentação por unidade de área, ocorre superestimativa do potencial de receita quando essas forrageiras são comparadas a outras de melhor valor nutritivo (silagem de milho). No entanto, estes resultados são obtidos em diferentes condições de utilização de concentrados e gastos com alimentação por unidade de área nas estratégias de alimentação (Tabela 1).

Entretanto, a metodologia ANUC permite condições de avaliação semelhantes em relação à utilização de concentrados e gastos com alimentação por área, entre os diferentes volumosos ou as estratégias de alimentação. Na realidade, o ponto de partida nas avaliações dessa metodologia é exatamente a utilização de concentrados e os gastos com alimentação por unidade de área, considerando que a prioridade é a melhor remuneração dos fatores de produção utilizados no sistema de produção de leite.

Na metodologia “Tradicional”, os resultados são comparados por nível de produção de leite por vaca, enquanto, na metodologia ANUC, os resultados são dependentes do nível de utilização de concentrados e gastos com alimentação por unidade de área. Nessa metodologia, o nível de produção de leite por vaca será dependente do valor nutritivo e das características agronômicas dos volumosos, em determinado nível de gastos com concentrados e alimentação por área, caracterizando múltiplas restrições.

A avaliação da estimativa da PL/vaca/ano, da mesma forma como nas avaliações da utilização de concentrados por área, demonstrou grandes diferenças nos resultados entre as estratégias de alimentação. Considerando todos os níveis de gastos com alimentação por área, as estratégias baseadas em silagem de milho e cana-de-açúcar, combina- 
das com pastagens, apresentaram, respectivamente, PL/vaca/ano de 97,11 e 62,61\% da observada na estratégia SIM (Tabela 6).

A maior PL/vaca/ano avaliada em determinado nível de gasto com alimentação/ha é fortemente influenciada pela qualidade das forrageiras que compõem a estratégia de alimentação. A utilização da silagem de milho, volumoso de melhor valor nutritivo, resultou nas maiores estimativas de produção de leite por vaca, independentemente do nível de gasto com alimentação por hectare. Como volumoso exclusivo ou em associação com pastagens, a silagem de milho apresentou os maiores valores estimados de produção individual de leite. A qualidade das pastagens e a capacidade de suporte das estratégias de alimentação também influenciaram os resultados. As estratégias com utilização de forrageiras de maior qualidade e de menor capacidade de suporte, representadas nesse estudo pelo capim-tifton 85, apresentaram as maiores médias estimadas de PL/vaca/ha, associado à silagem de milho ou à canade-açúcar (Tabela 6).

\section{Conclusões}

A utilização de concentrados e gastos com alimentação por unidade de área quando utilizada a metodologia de avaliação “Tradicional” resulta em grandes diferenças nos gastos dos fatores de produção comprometendo as avaliações da produtividade e da receita por unidade de área. A metodologia alternativa de avaliação ANUC compara as diferentes estratégias de alimentação, em mesma base de uso de fatores de produção, às estratégias de alimentação com utilização de volumosos de melhor qualidade, comprovando maior produtividade e renda por unidade de área. O nível de produção de leite por vaca entre as estratégias de alimentação difere quando fixadas as quantidades dos fatores de produção de leite por unidade de área quando utilizada a metodologia alternativa ANUC.

\section{Literatura Citada}

ASSIS, A.G.; BROCKINGTON, N.R. Sistemas de produção e econômia: o estado da arte. In: REUNIÃO ANUAL DA SOCIEDADE BRASILEIRA DE ZOOTECNIA, 33., 1995, Brasília. Anais... Brasília: Sociedade Brasileira de Zootecnia, 1995, p.573-582.

BATH, D.L.; SOSNIK, U. Formulation, delivery and inventory control of cost-effective rations. In: Van HORN, H.H.; WILCOX, C.J. (Eds.) Large dairy herd management. Savoy: American Dairy Science Association, 1992. p.709-719.

COSTA, M.G.; CAMPOS, J.M.S.; VALADARES FILHO, S.C. et al. Desempenho produtivo de vacas leiteiras alimentadas com diferentes proporções de cana-de-açúcar e concentrado ou silagem de milho na dieta. Revista Brasileira de Zootecnia, v.34, n.6, p.2437-2445, 2005 (supl.).

ELY, L.O. Economics of forage programs. In: In: VAN HORN, H.H.; WILCOX, C.J. (Eds.) Large dairy herd management. Savoy: American Dairy Science Association, 1992. p.720-730.

FERREIRA, A.H. Eficiência de sistemas de produção de leite: uma aplicação da análise envoltória de dados na tomada de decisão. Viçosa, MG: Universidade Federal de Viçosa, 2002. 120p. Dissertação (Mestrado em Economia Rural) - Universidade Federal de Viçosa, 2002.

FOX, D.G.; TYLUTKI, T.P.; TEDESCHI, L.O. et al. The net carbohydrate and protein system for evaluating herd nutrition and nutrient excretion. CNCPS version 5.0. Ithaca: Cornell University, 2003. 292p. (Model documentation).

NATIONAL RESEARCH COUNCIL - NRC. Nutrient requirements of dairy cattle. 6.ed. Washington, D.C.: National Academic Press, 1988. 157p.

NATIONAL RESEARCH COUNCIL - NRC. Nutrient requirements of dairy cattle. 7.ed. Washinton, D.C.: National Academic Press, 2001. 381p.

NOGUEIRA, M.P. Pontos para o bom gerenciamento de uma fazenda leiteira. [S.L.]: Scott Consultoria, 2004a. p.5-7 (A Nata do Leite, 76).

NOGUEIRA, M.P. Produção de volumosos: condução técnica e custos. [S.L.]: Scott Consultoria, 2004b. p.4-5 (A Nata do Leite, 78).

NUSSIO, L.G.; LIMA, L.G.; MATTOS, W.R.S. Alimentos volumosos para o período da seca. In: SIMPÓSIO SOBRE MANEJO E NUTRIÇÃO DE GADO DE LEITE, 2001, Goiânia. Anais.. Goiânia: CBNA, 2001. p.85-100.

NUSSIO, L.G.; LIMA, L.G.; MATTOS, W.R.S. Planejamento da produção de alimentos para o inverno. In: SIMPOSIO SOBRE A PRODUÇÃO ANIMAL, 10., 1998, Piracicaba. Anais... Piracicaba: Fundação de Estudos Agrários Luiz de Queiroz, 1998. p.57-94.

NUSSIO, L.G.; NUSSIO, C.M.B. Aspectos técnicos e econômicos que afetam a escolha da fonte de forragem suplementar. In: SIMPÓSIO INTERNACIONAL DE PRODUÇÃO INTENSIVA DE LEITE - INTERLEITE, 6., 2003, Uberaba. Anais... Uberaba: FMVZ-USP, 2003. p.123-135

OLIVEIRA, A.S.; CAMPOS, J.M.S.; GOMES, S.T. et al. Análise econômica e de sensibilidade da substituição da silagem de milho pela cana-de-açúcar corrigida em dietas de vacas leiteiras. In: REUNIÃO ANUAL DA SOCIEDADE BRASILEIRA DE ZOOTECNIA, 41., 2004, Campo Grande. Anais... Campo Grande:Gmosis, 2004. (CD-ROM).

PEREIRA, J.C. Vacas leiteiras: aspectos práticos da alimentação. Viçosa, MG: Aprenda Fácil, 2000. 198p.

RENNÓ, F.P.; PEREIRA, J.C.; LEITE, C.A.M. et al. Avaliação bioeconômica de estratégias de alimentação em sistemas de produção de leite 1. Produção por animal e por área. Revista Brasileira de Zootecnia, v.35, n.4, p.****_****, 2008 .

STATISTICAL ANALYSIS SYSTEM - SAS. SAS system user's guide - Online Doc. v.8.0, Cary: 1999. (CD-ROM).

TEDESCHI, L.O.; FOX, D.G.; PELL, A.N. et al. Development and evaluation of a tropical feed library for the Cornell Net Carbohydrate and Protein System model. Scientia Agricola, v.59, p.1-18, 2002.

UNDERSANDER, D.J.; HOWARD, W.T.; SHEWER, R.D. Milk per acre spreadsheet for combining yield and quality into a single term. Journal of Production Agriculture, v.2, p.231-235, 1993.

VALADARES FILHO, S.C.; ROCHA JR., V.R.; CAPPELLE, E.R. Tabelas brasileiras de composição de alimentos para bovinos. Viçosa, MG: Suprema Gráfica, 2002. 297p. 